\title{
An extension of Turán's Theorem, uniqueness and stability
}

\author{
Peter Allen* Julia Böttcher ${ }^{\dagger}$ \\ Department of Mathematics \\ London School of Economics \\ Houghton Street \\ London, WC2A 2AE, UK. \\ \{p.d.allen, j.boettcher\}@lse.ac.uk
}

\author{
Jan Hladký \\ Mathematics Institute \\ Czech Academy of Sciences \\ Žitná 25 \\ Praha, Czech Republic. \\ honzahladky@gmail.com
}

\author{
Diana Piguet ${ }^{\S}$ \\ European Centre of Excellence NTIS \\ University of West Bohemia \\ Univerzitní 8 \\ 30614 Pilsen, Czech Republic. \\ piguet@ntis.zcu.cz
}

Submitted: Mar 16, 2014; Accepted: Sep 23, 2014; Published: Oct 2, 2014

Mathematics Subject Classifications: 05C35

\begin{abstract}
We determine the maximum number of edges of an $n$-vertex graph $G$ with the property that none of its $r$-cliques intersects a fixed set $M \subseteq V(G)$. For $(r-1)|M| \geqslant$ $n$, the $(r-1)$-partite Turán graph turns out to be the unique extremal graph. For $(r-1)|M|<n$, there is a whole family of extremal graphs, which we describe explicitly. In addition we provide corresponding stability results.
\end{abstract}

*Supported by DIMAP, EPSRC award EP/D063191/1, FAPESP (Proc. 2010/09555-7) and grateful to NUMEC/USP, Núcleo de Modelagem Estocástica e Complexidade of the University of São Paulo, for supporting this research.

${ }^{\dagger}$ Supported by FAPESP (Proc. 2009/17831-7) and grateful to NUMEC/USP, Núcleo de Modelagem Estocástica e Complexidade of the University of São Paulo, for supporting this research.

${ }_{\ddagger}^{\ddagger}$ Supported by DIMAP, EPSRC award EP/D063191/1. The work was done while the author was an EPSRC Fellow at the Mathematics Institute, University of Warwick, UK.

${ }^{\S}$ Supported by DIMAP, EPSRC award EP/D063191/1. The research leading to this result has received funding from the European Union's Seventh Framework Programme (FP7/2007-2013) under grant agreement no. PIEF-GA-2009-253925. 


\section{Introduction}

Turán's Theorem [9], whose proof marks the beginning of Extremal Graph Theory, determines the maximum number of edges of $n$-vertex graph without a copy of the $r$-clique $K_{r}$. It turns out that the unique extremal graph for this problem is the Turán graph $\mathrm{T}_{r-1}(n)$, that is, the complete balanced $(r-1)$-partite graph on $n$-vertices. We write $t_{r-1}(n)$ to denote the number of edges of $\mathrm{T}_{r-1}(n)$.

Turán's Theorem is a primal example of a stable result: The Erdős-Simonovits stability theorem $[4,8]$ asserts that any $n$-vertex $K_{r}$-free graph with almost $t_{r-1}(n)$ edges looks very similar to $\mathrm{T}_{r-1}(n)$. In order to make this more precise we need the following definition. We say that an $n$-vertex graph $G$ is $\varepsilon$-close to a graph $H$ on the same vertex set if $H$ can be obtained from $G$ by editing (deleting/inserting) at most $\varepsilon n^{2}$ edges and relabelling the vertices. In this case we also say that $G$ is $\left(\varepsilon n^{2}\right)$-near to $H$.

Theorem 1 (Erdös [4] \& Simonovits [8]). Suppose that $r \geqslant 3$ and $\varepsilon^{*}>0$ are given. Then there exists $\gamma^{*}>0$ such that each $\ell$-vertex graph $G$ with no $K_{r}$ and $e(G)>t_{r-1}(\ell)-\gamma^{*} \ell^{2}$ is $\varepsilon^{*}$-close to $\mathrm{T}_{r-1}(\ell)$.

In fact, Erdős and Simonovits both proved more general statements, allowing any fixed $r$-partite graph $H$ in place of $K_{r}$. Moreover, in more recent years strengthenings have been proved, for example that most vertices of any $G$ as in Theorem 1 are in an induced $(r-1)$-partite graph [6]. There are also further generalisations, such as obtaining the same conclusion as in Theorem 1 while allowing the size of the forbidden subgraph $H$ to depend on $v(G)[7]$.

A main motivation for proving stability results for extremal statements is that they are often useful in applications where the original extremal statement would not suffice. This is for example the case when the Szemerédi Regularity Lemma (see, e.g., the survey [5]) is used. A prominent example of such an application is the enumeration result of Balogh, Bollobás and Simonovits [3] giving a precise count of $H$-free graphs. It is worth observing that in most applications the 'basic' stability theorem of Erdös and Simonovits, Theorem 1, suffices.

Our goal is to extend Turán's Theorem, by determining the maximum number of edges in an $n$-vertex graph $G$ such that no copy of $K_{r}$ in $G$ touches a fixed vertex set $M \subseteq V(G)$ of size $m$. It turns out that for $(r-1) m \geqslant n$ the unique extremal graph is $\mathrm{T}_{r-1}(n)$. The case $(r-1) m<n$ is more complicated. In particular, there is a whole family of extremal graphs, which we describe in Section 1.1 below. In both cases we shall denote the (family of) extremal graphs by $\mathcal{T}_{r-1}(n, m)$, and their number of edges by

$$
t_{r-1}(n, m):= \begin{cases}t_{r-1}(n), & \text { if } n \leqslant(r-1) m \\
\left(\begin{array}{c}
n \\
2
\end{array}\right)-n m+(r-1)\left(\begin{array}{c}
m+1 \\
2
\end{array}\right), & \text { otherwise } .\end{cases}
$$

Our main result is as follows.

Theorem 2. Given $r \geqslant 3$ and $m \leqslant n$, let $G$ be any $n$-vertex graph and $M \subseteq V(G)$ contain $m$ vertices, such that no copy of $K_{r}$ in $G$ intersects $M$. Then 
(a) $e(G) \leqslant t_{r-1}(n, m)$, and

(b) if $e(G)=t_{r-1}(n, m)$ then $G \in \mathcal{T}_{r-1}(n, m)$.

Moreover, given $\varepsilon>0$, there exists $\gamma>0$ such that the following holds.

(c) If $e(G)>t_{r-1}(n, m)-\gamma n^{2}$, then $G$ is $\varepsilon$-close to a graph from $\mathcal{T}_{r-1}(n, m)$ in which no copies of $K_{r}$ intersect $M$.

Theorem 2(b) states that the graphs $\mathcal{T}_{r-1}(n, m)$ we construct below are the only extremal graphs, while $(c)$ asserts stability. We remark that Theorem $2(a)$ is also included in our previous paper [2], but we did not determine the family of extremal graphs there. Hence our main contribution here is to determine the extremal graphs and prove stability. This, however, turns out to be an important tool for [1], where we determine the maximum number of edges in an $n$-vertex graph without a given number of vertex-disjoint triangles. Note that the statement of Theorem 2(c) gives a slighly stronger version of stability than the usual one, namely that the set $M$ is not changed in transforming $G$ to a member of $\mathcal{T}_{r-1}(n, m)$. We require this in [1].

We note that the proof of Theorem $2(a)$ as given in [2] hints the main arguments involved in our proof of Theorem 2. However, several additional tweaks and tricks are needed, in particular in the case $n>(r-1) m$.

The $(r-1) m \geqslant n$ case of Theorem 2 shows that the assumption in Turán's Theorem (or in that of Theorem 1) can be substantially weakened from forbidding $K_{r}$-copies on all possible $r$-subsets of the vertex set $V(G)$, to just forbidding $K_{r}$-copies on a particular family $\mathcal{S}$ of $r$-subsets - the family $\mathcal{S}$ which contains all $r$-subsets of $V(G)$ which intersect $M$. In [2] we investigated such weakenings of the assumption in Turán's theorem also from a probabilistic perspective. In particular, we proved that forbidding $K_{r}$-copies on a random family of $r$-sets $\mathcal{S} \subseteq\left(\begin{array}{l}n \\ r\end{array}\right)$ of size only $|\mathcal{S}|=\mathcal{O}\left(n^{3}\right)$ suffices.

\subsection{Extremal graphs}

The family $\mathcal{T}_{r-1}(n, m)$ is defined as follows. As previously stated, if $n \leqslant(r-1) m$ then $\mathcal{T}_{r-1}(n, m)=\left\{\mathrm{T}_{r-1}(n)\right\}$. So assume from now on that $n>(r-1) m$. We explicitly describe the construction of the graphs in $\mathcal{T}_{r-1}(n, m)$.

We start with the Turán graph $\mathrm{T}_{r-1}((r-1) m)$, with colour classes $V_{1}, \ldots, V_{r-1}$, and an arbitrary set $M$ of $m$ vertices in $V_{1} \cup \cdots \cup V_{r-1}$. We add $r-1$ new vertices $v_{1}, \ldots, v_{r-1}$ to this graph with the following property. For each $i \in[r-1]$, the vertex $v_{i}$ is adjacent to all old and new vertices except those in $V_{i}$ (and itself). Finally, we add a set $Y$ of $n-(r-1) m$ new vertices each of which is adjacent to all old and new vertices except those in $M$ (and itself). In this way we obtain an $(n+r-1)$-vertex graph, which we call $G_{r}(n, M)$. Note that the graph $G_{r}(n, M)$ depends on the placement of $M$ in $V_{1} \cup \cdots \cup V_{r-1}$. We let $\mathcal{T}_{r-1}(n, m)$ be the family of $n$-vertex graphs which can be obtained from some graph $G_{r}(n, M)$ by deleting any $r-1$ vertices from $\left\{v_{1}, \ldots, v_{r-1}\right\} \cup Y$ (see also Figure 1.1). We call the vertices $v_{1}, \ldots, v_{r-1}$ sporadic. 

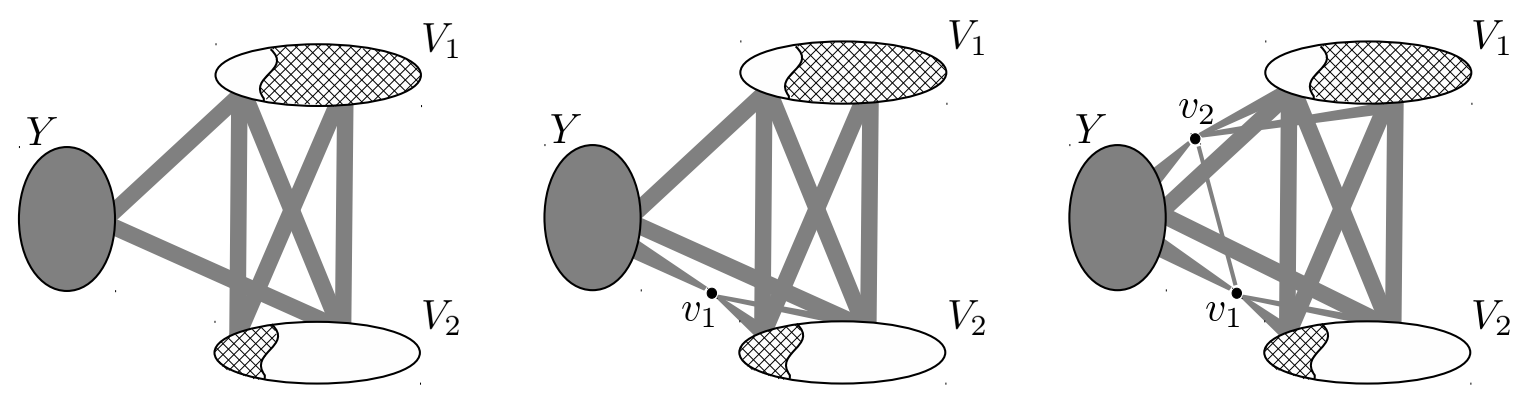

Figure 1: Examples of graphs from $\mathcal{T}_{2}(n, m)$ for $n>2 m$, with no, one, and two sporadic vertices. Grey depicts complete (bipartite) graphs, the set $M$ is hatched.

Observe that there is no copy of $K_{r}$ in $G_{r}(n, M)$ which uses vertices of $M$. Furthermore, the vertices $\left\{v_{1}, \ldots, v_{r-1}\right\} \cup Y$ form a clique in $G_{r}(n, M)$, and each of these vertices has degree $n+r-2-m$. It follows that indeed every graph in $\mathcal{T}_{r-1}(n, m)$ has the same number of edges, and that number is

$$
\left(\begin{array}{c}
n-(r-1) m \\
2
\end{array}\right)+(n-(r-1) m)(r-2) m+t_{r-1}((r-1) m)=t_{r-1}(n, m),
$$

as desired.

\section{Proof of Theorem 2}

We refer to the cases $n \leqslant(r-1) m$ and $n>(r-1) m$ as Cases I and II, respectively. We prove Case I first, and then prove Case II using Case I.

\subsection{Case I}

The following lemma will be the key tool for proving uniqueness and stability when $n \leqslant(r-1) m$.

Lemma 3. Given $m$ and $n \leqslant(r-1) m$, let $G$ be an $n$-vertex graph and $M$ a subset of $V(G)$ with $|M|=m$ such that no copy of $K_{r}$ in $G$ uses vertices of $M$. Suppose that there are sets $P_{1}, \ldots, P_{k}$ of sizes $p_{1}, \ldots, p_{k}$ in $G$ such that the following holds for all $i \in[k]$.

(i) $\left|P_{i}\right| \geqslant r$.

(ii) $P_{i}$ is the vertex set of a maximum clique in $G\left[V(G) \backslash \cup_{j=1}^{i-1} P_{j}\right]$.

(iii) $G\left[V(G) \backslash \cup_{j=1}^{k} P_{j}\right]$ contains no $K_{r}$.

Let $p:=\sum_{\ell=1}^{k} p_{\ell}$. Then we have

$$
e(G) \leqslant t_{r-1}(n)-\sum_{i=1}^{k} \sum_{j=0}^{p_{i}-r}\left(m-\left\lfloor\frac{n-\left(p-j-\sum_{\ell=i+1}^{k} p_{\ell}\right)}{r-1}\right\rfloor-1\right) .
$$


Proof. We first establish some simple bounds on the number of edges in $G$. Each $P_{i}$ contains $\left(\begin{array}{c}p_{i} \\ 2\end{array}\right)$ edges. By the maximality of $P_{1}, \ldots, P_{k}$ we have $\operatorname{deg}\left(v, P_{i}\right) \leqslant p_{i}-1$ for any $v \in V(G) \backslash \bigcup_{j=1}^{i} V\left(P_{i}\right)$. Because no copy of $K_{r}$ in $G$ intersects $M$, we have $M \subseteq$ $V(G) \backslash \bigcup_{i=1}^{k} V\left(P_{i}\right)$ and the stronger $\operatorname{bound} \operatorname{deg}\left(v, P_{i}\right) \leqslant r-2$ for each $v \in M$. Finally, since the graph $G-\cup_{i=1}^{k} P_{i}$ is $K_{r}$-free, by Turán's theorem we have

$$
e\left(G-\bigcup_{i=1}^{k} V\left(P_{i}\right)\right) \leqslant t_{r-1}(n-p) .
$$

Putting these estimates together we obtain

$$
\begin{aligned}
e(G) \leqslant \sum_{i=1}^{k}\left(\begin{array}{c}
p_{i} \\
2
\end{array}\right) & +\sum_{1 \leqslant i<j \leqslant k}\left(p_{i}-1\right) p_{j}+(p-k)(n-m-p) \\
& +m k(r-2)+t_{r-1}(n-p) .
\end{aligned}
$$

Observe that the right hand side of (3) defines a function, which we denote $g_{n}\left(p_{1}, \ldots, p_{k}\right)$, whose domain is the set of tuples (of any length $k$ ) of nonnegative integers. In particular we allow $k=0$, when $(3)$ gives $g_{n}()=t_{r-1}(n)$.

We now give two equalities relating values of $g_{n}$. As a preparatory step, observe that for any $n^{\prime}$ we have

$$
\begin{aligned}
& t_{r-1}\left(n^{\prime}+1\right)-t_{r-1}\left(n^{\prime}\right)=n^{\prime}-\left\lfloor\frac{n^{\prime}}{r-1}\right\rfloor, \quad \text { and } \\
& t_{r-1}\left(n^{\prime}+r\right)-t_{r-1}\left(n^{\prime}\right)=(r-1) n^{\prime}+\left(\begin{array}{l}
r \\
2
\end{array}\right)-\left\lfloor\frac{n^{\prime}+r-1}{r-1}\right\rfloor .
\end{aligned}
$$

Now suppose that $k \geqslant 1$. If $p_{k}>r$ then plugging (4) (with $n^{\prime}=n-p=n-\sum_{\ell=1}^{k} p_{\ell}$ ) into the definition of $g_{n}$ in (3) we obtain

$$
g_{n}\left(p_{1}, \ldots, p_{k-1}, p_{k}-1\right)-g_{n}\left(p_{1}, \ldots, p_{k-1}, p_{k}\right)=m-\left\lfloor\frac{n-\sum_{\ell=1}^{k} p_{\ell}}{r-1}\right\rfloor-1 .
$$

Similarly, if $p_{k}=r$ then (5) implies

$$
g_{n}\left(p_{1}, \ldots, p_{k-1}\right)-g_{n}\left(p_{1}, \ldots, p_{k-1}, p_{k}\right)=m-\left\lfloor\frac{n-\sum_{\ell=1}^{k} p_{\ell}}{r-1}\right\rfloor-1 .
$$

We note that our condition $n \leqslant(r-1) m$ implies that $m-\left\lfloor\frac{n-p}{r-1}\right\rfloor-1>0$.

Applying repeatedly both (6) and (7) we obtain

$$
g_{n}()-g_{n}\left(p_{1}, \ldots, p_{k}\right)=\sum_{i=1}^{k} \sum_{j=0}^{p_{i}-r}\left(m-\left\lfloor\frac{n-\left(p-j-\sum_{\ell=i+1}^{k} p_{\ell}\right)}{r-1}\right\rfloor-1\right),
$$

which together with $e(G) \leqslant g_{n}\left(p_{1}, \ldots, p_{k}\right)$ and $g_{n}()=t_{r-1}(n)$ yields the desired bound on $e(G)$. 
We are now ready to prove Theorem 2 in Case I.

Proof of Theorem 2, Case I. Let $G$ be an $n$-vertex graph and $M$ a subset of $V(G)$ of size $m$, where $n \leqslant(r-1) m$, such that no $K_{r}$ of $G$ intersects $M$. We iteratively find vertex disjoint cliques $P_{1}, \ldots, P_{k}$ of sizes $p_{1}, \ldots, p_{k}$ with at least $r$ vertices as follows. Suppose that for some $i$, the cliques $P_{1}, \ldots, P_{i-1}$ have already been defined. Let $P_{i}$ be an arbitrary maximum clique on at least $r$ vertices in the graph $G-\bigcup_{j<i} P_{j}$. We set $k:=i-1$ and terminate if no such clique exists. Let $p:=\sum_{\ell=1}^{k} p_{\ell}$. Now $G, M$ and $P_{1}, \ldots, P_{k}$ satisfy the conditions of Lemma 3, so we have

$$
e(G) \leqslant t_{r-1}(n)-\sum_{i=1}^{k} \sum_{j=0}^{p_{i}-r}\left(m-\left\lfloor\frac{n-\left(p-j-\sum_{\ell=i+1}^{k} p_{\ell}\right)}{r-1}\right\rfloor-1\right) .
$$

We first prove the claimed bound $(a)$ and uniqueness $(b)$. We distinguish two cases. First, $G$ contains no copy of $K_{r}$. In this case Turán's theorem guarantees that $e(G) \leqslant$ $t_{r-1}(n)$ with equality if and only if $G=\mathrm{T}_{r-1}(n)$.

Second, $G$ contains at least one copy of $K_{r}$. In this case, there is at least one term in the double sum in (8) (since $P_{1}$ exists) and the smallest of the summands is that with $i=1$ and $j=p_{1}-r$, i.e.,

$$
m-\left\lfloor\frac{n-\left(p-\left(p_{1}-r\right)-\sum_{\ell=2}^{k} p_{\ell}\right)}{r-1}\right\rfloor-1=m-\left\lfloor\frac{n-r}{r-1}\right\rfloor-1=m-\left\lfloor\frac{n-1}{r-1}\right\rfloor .
$$

Since $n \leqslant(r-1) m$, we have $m \geqslant\left\lceil\frac{n}{r-1}\right\rceil$ and hence the smallest summand is at least 1 . It follows that $e(G)<t_{r-1}(n)$ and so $G$ is not extremal. This proves $(a)$ and $(b)$.

It remains to prove part $(c)$. Given $\varepsilon>0$, we let $\gamma^{*}$ be the constant given by Theorem 1 for the input $\varepsilon^{*}:=\varepsilon / 2$. We let

$$
\gamma_{1}:=\min \left(\gamma^{*}, \frac{1}{4}, \varepsilon\right) \quad \text { and } \quad \gamma:=\frac{\gamma_{1}^{2}}{64 r^{2}} .
$$

Suppose that $e(G) \geqslant t_{r-1}(n, m)-\gamma n^{2}$. We may assume that $\gamma n^{2} \geqslant 1$, as otherwise our uniqueness result gives $G=\mathrm{T}_{r-1}(n)$. It follows in particular by (9) that $\gamma_{1} n \geqslant 8 r$, which in turn gives

$$
p-2 r \geqslant p-\gamma_{1} n / 4 .
$$

Observe that the $p-(k-1) r$ values $j+\sum_{\ell=i+1}^{k} p_{\ell}$ in $(8)$ form a sequence of distinct integers, with, if ordered, consecutive values separated by either 1 or $r$, and the smallest is 0 . Thus at least $p /(2 r)$ of these values satisfy $j+\sum_{\ell=i+1}^{k} p_{\ell} \leqslant p / 2$, or equivalently, $p-j-\sum_{\ell=i+1}^{k} p_{\ell} \geqslant p / 2$. In addition, as before all summands of the double sum in (8) are non-negative. It follows that

$$
\begin{aligned}
e(G) & \leqslant t_{r-1}(n)-\frac{p}{2 r}\left(m-\left\lfloor\frac{n-p / 2}{r-1}\right\rfloor-1\right) \\
& \leqslant t_{r-1}(n)-\frac{p}{2 r}\left(m-\frac{n}{r-1}+\frac{p-2 r}{2 r-2}\right) \leqslant t_{r-1}(n)-\frac{p(p-2 r)}{4 r^{2}}
\end{aligned}
$$


where we used $n \leqslant(r-1) m$ in the last inequality. Since $e(G) \geqslant t_{r-1}(n)-\gamma n^{2}$, we can use (9) and (10) to conclude $p \leqslant \gamma_{1} n / 2$.

Let $G^{\prime}$ be the subgraph of $G$ induced by $V(G) \backslash \cup_{i=1}^{k} P_{i}$. We have

$$
e\left(G^{\prime}\right)>t_{r-1}(n)-\gamma n^{2}-\frac{1}{2} \gamma_{1} n^{2} \stackrel{(9)}{\geqslant} t_{r-1}(n)-\frac{3}{4} \gamma_{1} n^{2}
$$

and since $v\left(G^{\prime}\right) \geqslant\left(1-\gamma_{1} / 2\right) n \stackrel{(9)}{\geqslant} \frac{7}{8} n$, we have $e\left(G^{\prime}\right)>t_{r-1}\left(v\left(G^{\prime}\right)\right)-\gamma_{1} v\left(G^{\prime}\right)^{2}$. By definition of the sets $P_{i}$ the graph $G^{\prime}$ is $K_{r}$-free. Therefore, by Theorem 1 the graph $G^{\prime}$ is $\varepsilon^{*}$-close to $\mathrm{T}_{r-1}\left(v\left(G^{\prime}\right)\right)$. It follows that $G$ is $\left(\varepsilon^{*} v\left(G^{\prime}\right)^{2}+\gamma_{1} n^{2} / 2\right)$-near to $\mathrm{T}_{r-1}(n)$, and thus by $(9)$ that $G$ is $\varepsilon$-close to $\mathrm{T}_{r-1}(n)$ as required.

\subsection{Case II}

We first state three lemmas which we will use to prove Theorem 2 in Case II. Note that the first two of these lemmas do not require the condition $n>(r-1) m$. The first lemma asserts that every graph $G$ with no $K_{r}$ intersecting $M$ can easily be modified such that each vertex outside $M$ has high degree.

Lemma 4. Let $G$ be an n-vertex graph and $M \subseteq V(G)$ have size $m$. Assume that no copy of $K_{r}$ in $G$ intersects $M$. Given $\mu \in[0,1)$, there is a graph $G^{\prime}$ on $V(G)$ with the following properties.

(a) $G^{\prime}$ has no copy of $K_{r}$ intersecting $M$.

(b) $e\left(G^{\prime}\right) \geqslant e(G)$, with equality if and only if $G=G^{\prime}$.

(c) Either $e\left(G^{\prime}\right)>e(G)+\mu^{2} n^{2}$, or $G^{\prime}$ is $\mu n^{2}$-near to $G$ (without relabelling vertices).

(d) Every vertex $v \in V(G) \backslash M$ has $\operatorname{deg}_{G^{\prime}}(v) \geqslant n-m-\mu n-1$.

Proof. We obtain $G^{\prime}$ from $G$ by repeating the following procedure until conclusion $(d)$ is satisfied. If there exists a vertex $v \in V(G) \backslash M$ with degree smaller than $n-m-\mu n-1$, delete all edges containing $v$ and insert all edges from $v$ to $V(G) \backslash(M \cup\{v\})$.

Observe that at each step, we add at least $\mu n$ edges to the graph, and edit at most $n$ edges. It follows that the algorithm terminates, and thus conclusions $(b)$ and $(d)$ get satisfied. Clearly, the resulting graph $G$ also satisfies $(a)$. Furthermore, if the procedure is repeated more than $\mu n$ times, then $e\left(G^{\prime}\right)-e(G)>\mu^{2} n^{2}$, while otherwise the number of edits is at most $\mu n^{2}$, so conclusion $(c)$ is satisfied.

The next lemma states that there are few vertices which have big degree in $G$ and many neighbours in $M$.

Lemma 5. Let $G$ be an n-vertex graph and $M \subseteq V(G)$ have size $m$. Assume that no copy of $K_{r}$ in $G$ intersects $M$. Given $\nu \in[0,1)$, let $X$ be the set of vertices in $G$ outside $M$ with at least $\max (1, \nu n)$ neighbours in $M$. Suppose that every vertex of $X$ has degree at least $n-m-\nu^{2} n$. Then we have $|X| \leqslant(1+\nu)(r-2) m$. 
Proof. Let $x_{1}, \ldots, x_{k}$ be the vertices of a maximum clique in $G[X]$. For each $i \in[k]$, let $s_{i}$ be the number of non-neighbours of $x_{i}$ in $X$ (including $x_{i}$ itself). Because $x_{1}, \ldots, x_{k}$ is a maximum clique, every vertex of $X$ is a non-neighbour of at least one $x_{i}$, and therefore we have $s_{1}+\ldots+s_{k} \geqslant|X|$.

Observe that $x_{i}$ has at most $n-m-s_{i}$ neighbours outside $M$. Hence, by definition of $X$ and since $\operatorname{deg}\left(x_{i}\right) \geqslant n-m-\nu^{2} n$ the vertex $x_{i}$ has at least $\max \left(\nu n, s_{i}-\nu^{2} n\right)$ neighbours in $M$. On the other hand, no vertex of $M$ is adjacent to more than $r-2$ of the vertices $x_{1}, \ldots, x_{k}$, or there would be a copy of $K_{r}$ intersecting $M$. It follows that $(r-2)|M| \geqslant k \nu n$ and

$$
(r-2)|M| \geqslant \sum_{i=1}^{k}\left(s_{i}-\nu^{2} n\right) \geqslant|X|-k \nu^{2} n \geqslant|X|-\nu(r-2)|M|,
$$

from which we have $|X| \leqslant(1+\nu)(r-2)|M|$.

The final preparatory lemma asserts that $\mathcal{T}_{r-1}(n, m)$ is closed under certain local modifications.

Lemma 6. Suppose that $n>(r-1) m$. Let $G_{1} \in \mathcal{T}_{r-1}(n, m)$ be a graph in which no $K_{r}$ intersects the $m$-set $M \subseteq V\left(G_{1}\right)$, and let $v \in V\left(G_{1}\right) \backslash M$ be a vertex whose neighbourhood in $G_{1}$ is $V\left(G_{1}\right) \backslash(M \cup\{v\})$. Delete all edges incident to $v$ and insert $n-m-1$ edges, of which at least one goes to $M$. If there is no copy of $K_{r}$ intersecting $M$ in the modified graph $G_{2}$, then $G_{2} \in \mathcal{T}_{r-1}(n, m)$.

Proof. Recall that since $G_{1}$ is in $\mathcal{T}_{r-1}(n, m)$, it contains a copy of $\mathrm{T}_{r-1}((r-1) m)$ with colour classes $V_{1}, \ldots, V_{r-1}$ which covers $M$, but which does not cover $v$ because each of its vertices is either in or adjacent to $M$ in $G_{1}$. The same sets $V_{1}, \ldots, V_{r-1}$ continue to induce a copy of $\mathrm{T}_{r-1}((r-1) m)$ in $G_{2}$. Since $v$ has at least one $G_{2}$-neighbour in $M$, we can let $w_{i}$ be a neighbour of $v$ in $M \cap V_{i}$ for some $i$. If $v$ is adjacent to at least one vertex of each set $V_{1}, \ldots, V_{r-1}$, then letting $w_{j}$ be a neighbour of $v$ in $V_{j}$ for each $j \neq i$, we obtain a copy of $K_{r}$ in $G_{2}$ intersecting $M$, which is a contradiction. Thus there is $j$ such that $v$ has no neighbours in $V_{j}$, and since $v$ has degree $n-m-1$ it follows that the neighbourhood of $v$ is precisely $V\left(G_{1}\right) \backslash\left(V_{j} \cup\{v\}\right)$. In other words, $v$ has the same neighbourhood as a sporadic vertex in our construction, and we need only to show that there is no second vertex $v^{\prime} \neq v$ with neighbourhood $V\left(G_{1}\right) \backslash\left(V_{j} \cup\left\{v^{\prime}\right\}\right)$. If such a vertex existed, then $v, v^{\prime}$ and $w_{i}$ together with one vertex in each set $V_{\ell}$ with $\ell \notin\{i, j\}$ would form a copy of $K_{r}$ intersecting $M$ in $G_{2}$.

We can now prove Case II of Theorem 2.

Proof of Theorem 2, Case II. Let $G=(V, E)$ and $M$ satisfy the conditions of the theorem. First we show that $e(G) \leqslant t_{r-1}(n, m)$, with equality only for graphs in $\mathcal{T}_{r-1}(n, m)$, which proves $(a)$ and $(b)$.

We apply Lemma 4 to $G$ with $\mu:=0$ to obtain a graph $G^{\prime}$ on $V$ which also has no $K_{r}$ intersecting $M$, which has $e\left(G^{\prime}\right) \geqslant e(G)$ with equality only if $G=G^{\prime}$, and which is such 
that every vertex $v \in V \backslash M$ has $\operatorname{deg}_{G^{\prime}}(v) \geqslant n-m-1$. We now apply repeatedly the following further transformation to $G^{\prime}$ to obtain $G^{\prime \prime}$. If there exists a vertex $v$ in $V \backslash M$ whose degree is $n-m-1$ and which has a neighbour in $M$, we delete all edges incident to $v$, and insert all edges from $v$ to $V \backslash(M \cup\{v\})$. Observe that $e\left(G^{\prime \prime}\right)=e\left(G^{\prime}\right)$, and $G^{\prime \prime}$ satisfies the conditions of Lemma 5 with $\nu:=0$. It follows that the set $X$ of $G^{\prime \prime}$-neighbours of $M$ in $V \backslash M$ has size $|X| \leqslant(r-2) m$. Let $X^{\prime}$ be a subset of $V \backslash M$ containing $X$ of size exactly $(r-2) m$.

Since $\left|X^{\prime} \cup M\right|=(r-1) m$, we can now apply assertions $(a)$ and $(b)$ in Case I to conclude that

$$
e\left(G^{\prime \prime}\left[X^{\prime} \cup M\right]\right) \leqslant t_{r-1}((r-1) m, m)=t_{r-1}((r-1) m)
$$

with equality only if $G^{\prime \prime}\left[X^{\prime} \cup M\right]=\mathrm{T}_{r-1}((r-1) m)$. Observe that the vertices in $V \backslash$ $\left(X^{\prime} \cup M\right)$ are all of degree $n-m-1$ and have no neighbours in $M$. It follows that $e\left(G^{\prime \prime}\right) \leqslant t_{r-1}(n, m)$, with equality only if $G^{\prime \prime} \in \mathcal{T}_{r-1}(n, m)$. Since $e(G) \leqslant e\left(G^{\prime}\right)=e\left(G^{\prime \prime}\right)$, we have $e(G) \leqslant t_{r-1}(m, n)$, with equality only if $G=G^{\prime}$ and $G^{\prime \prime} \in \mathcal{T}_{r-1}(n, m)$. It remains only to show that if $e(G)=t_{r-1}(n, m)$, then the transformation from $G=G^{\prime}$ to $G^{\prime \prime}$ cannot take a graph outside $\mathcal{T}_{r-1}(n, m)$ to a graph in $\mathcal{T}_{r-1}(n, m)$. Observe that the reverse of this transformation consists exactly of steps satisfying Lemma 6, which therefore asserts that since $G^{\prime \prime} \in \mathcal{T}_{r-1}(n, m)$, so $G=G^{\prime} \in \mathcal{T}_{r-1}(n, m)$. This proves assertions $(a)$ and $(b)$.

Finally, we prove stability, that is, assertion $(c)$. Given $\varepsilon>0$, set $\varepsilon^{\prime}:=\varepsilon / 2$. Let $\gamma^{\prime}$ be the constant returned by the previously proved stability result for input $\varepsilon^{\prime}$ and define

$$
\nu:=\gamma^{\prime} \varepsilon^{\prime 2} / 4, \quad \mu:=\nu^{2} / 2 \quad \text { and } \quad \gamma:=\mu^{2} / 2 .
$$

Suppose that $e(G) \geqslant t_{r-1}(n, m)-\gamma n^{2}$. If $\gamma n^{2}<1$, then $e(G)=t_{r-1}(n, m)$ and so $G \in \mathcal{T}_{r-1}(n, m)$ (and in particular $G$ is $\varepsilon$-close to a graph in $\mathcal{T}_{r-1}(n, m)$ ). It follows that we may assume $n \geqslant \gamma^{-1 / 2}$, and so by (11) that $\mu n \geqslant 1$.

We apply Lemma 4 to $G$ to obtain a graph $G^{\prime}$ in which no copy of $K_{r}$ intersects $M$, with $e\left(G^{\prime}\right) \geqslant e(G)$, and in which every vertex $v \in V \backslash M$ has $\operatorname{deg}_{G^{\prime}}(v) \geqslant n-m-\mu n-1$. In particular, we have $e\left(G^{\prime}\right) \leqslant t_{r-1}(n, m)$. Since $\mu^{2}>\gamma$ by $(11)$, we must have $e\left(G^{\prime}\right) \leqslant$ $e(G)+\mu^{2} n^{2}$, so by conclusion $(c)$ of Lemma 4 the graph $G^{\prime}$ is obtained from $G$ by editing at most $\mu n^{2}$ edges.

Now since $\mu n \geqslant 1$ and by (11), we have $\operatorname{deg}_{G^{\prime}}(v) \geqslant n-m-2 \mu n=n-m-\nu^{2} n$ for each $v \in V \backslash M$. Letting $X$ be the vertices in $V \backslash M$ with at least $\nu n$ neighbours in $M$, we obtain by Lemma 5 that $|X| \leqslant(1+\nu)(r-2) m$.

Let $X^{\prime}$ be a subset of $V \backslash M$ of size $(r-2) m$ which is either contained in $X$ (if $|X|>(r-2) m$ ) or contains $X$ (if $|X| \leqslant(r-2) m$ ). We obtain a graph $G^{\prime \prime}$ by deleting all edges from $V \backslash\left(M \cup X^{\prime}\right)$ to $M$. Observe that, since $(r-2) m<n$, the graph $G^{\prime \prime}$ is obtained from $G^{\prime}$ by deleting at most $(n-m-|X|) \nu n+\nu(r-2) m^{2} \leqslant 2 \nu n^{2}$ edges, and therefore has $e\left(G^{\prime \prime}\right) \geqslant e\left(G^{\prime}\right)-2 \nu n^{2} \geqslant t_{r-1}(n, m)-\gamma n^{2}-2 \nu n^{2}$ edges. Furthermore, no copy of $K_{r}$ in $G^{\prime \prime}$ intersects $M$.

Let $H=G^{\prime \prime}\left[X^{\prime} \cup M\right]$. Since there are no edges in $G^{\prime \prime}$ between $V \backslash\left(X^{\prime} \cup M\right)$ and $M$, 
we have

$$
\begin{aligned}
e\left(G^{\prime \prime}\right) & =e\left(G^{\prime \prime}\left[V \backslash\left(X^{\prime} \cup M\right)\right]\right)+e\left(G^{\prime \prime}\left[V \backslash\left(X^{\prime} \cup M\right), X^{\prime} \cup M\right]\right)+e(H) \\
& \leqslant\left(\begin{array}{c}
n-(r-1) m \\
2
\end{array}\right)+(n-(r-1) m)(r-2) m+e(H) .
\end{aligned}
$$

Thus $e(H) \geqslant t_{r-1}((r-1) m)-\gamma n^{2}-2 \nu n^{2}$. Furthermore, by $(a)$ of Case I, $e(H) \leqslant$ $t_{r-1}((r-1) m)$.

We distinguish two cases. First, $(r-1) m \geqslant \varepsilon^{\prime} n$. In this case, we have

$$
e(H) \geqslant t_{r-1}((r-1) m)-\frac{\gamma+2 \nu}{\varepsilon^{\prime 2}}(r-1)^{2} m^{2} \stackrel{(11)}{\geqslant} t_{r-1}((r-1) m)-\gamma^{\prime}(r-1)^{2} m^{2} .
$$

We apply $(c)$ of Case I to $H$ with $\gamma^{\prime}$ and $\varepsilon^{\prime}$, to obtain that $H$ is $\varepsilon^{\prime}$-close to $\mathrm{T}_{r-1}((r-1) m)$. Second, $(r-1) m<\varepsilon^{\prime} n$. In this case, we have $\left(\begin{array}{c}(r-1) m \\ 2\end{array}\right)<\varepsilon^{\prime} n^{2}$.

We can thus, in either case, edit at most $\varepsilon^{\prime} n^{2}$ edges of $G^{\prime \prime}$ to obtain a graph $G^{\prime \prime \prime}$ in which $G^{\prime \prime \prime}\left[X^{\prime} \cup M\right]$ is a copy of $\mathrm{T}_{r-1}((r-1) m)$. Clearly, $G^{\prime \prime \prime}$ is a subgraph of a graph in $\mathcal{T}_{r-1}(n, m)$ (without sporadic vertices), and $e\left(G^{\prime \prime \prime}\right) \geqslant e\left(G^{\prime \prime}\right) \geqslant t_{r-1}(n, m)-\gamma n^{2}-2 \nu n^{2}$. It follows that we can add at most $\gamma n^{2}+2 \nu n^{2}$ edges to $G^{\prime \prime \prime}$ to obtain a graph $T$ in $\mathcal{T}_{r-1}(n, m)$. In total, we have made

$$
\mu n^{2}+2 \nu n^{2}+\varepsilon^{\prime} n^{2}+\gamma n^{2}+2 \nu n^{2} \stackrel{(11)}{\leqslant} \varepsilon n^{2}
$$

edits from $G$ to $T$, and have preserved the property that no copy of $K_{r}$ intersects $M$.

\section{Concluding remarks}

Theorem 2 forbids $K_{r}$-copies which intersect $M$. An obvious extension would be to forbid $K_{r}$-copies which intersect $M$ in at least $s$ vertices. We suspect that, at least for small $s$, similar methods to those used here might give corresponding results also for this setting.

Another possible direction of extending Theorem 2 is to forbid a general fixed $r$-partite graph $H$, instead of $K_{r}$, to touch the set $M$. The standard regularity method allows one to deduce that the upper bound from Theorem 2(a) holds even in this case, up to an additive $o\left(n^{2}\right)$ term. The Turán graph provides an almost matching lower bound in Case I, and the regularity method proves the corresponding stability statement in this case. In Case II, however, the graphs in $\mathcal{T}_{r-1}(n, m)$ do not necessarily provide a lower bound. For example, each of the graphs in $\mathcal{T}_{2}(n, m)$ contains a copy of $C_{5}$ touching the set $M$. It would be interesting to determine the true extremal results in such cases.

Finally, one could ask for a stronger stability result in the spirit of [6]. This can be obtained easily from Theorem $2(c)$ as follows. We take the graph $G^{\prime}$ in $\mathcal{T}_{r-1}(n, m)$ close in edit distance to $G$, and delete all vertices whose neighbourhoods in $G^{\prime}$ and $G$ have a large enough symmetric difference. There can only be few such vertices by definition, and it is now easy to check that any remaining edge between $M$ and $Y$, or within a part $V_{i}$, can be extended to a copy of $K_{r}$ in $G$ touching $M$. 


\section{References}

[1] P. Allen, J. Böttcher, J. Hladký, and D. Piguet, A density Corrádi-Hajnal theorem, Canadian Journal of Mathematics, to appear, doi :10.4153/CJM-2014-030-6.

[2] P. Allen, J. Böttcher, J. Hladký, and D. Piguet, Turánnical hypergraphs, Random Structures Algorithms 42 (2013), no. 1, 29-58.

[3] József Balogh, Béla Bollobás, and Miklós Simonovits, The number of graphs without forbidden subgraphs, J. Combin. Theory Ser. B 91 (2004), no. 1, 1-24.

[4] P. Erdős, On some new inequalities concerning extremal properties of graphs, Theory of Graphs (Proc. Colloq., Tihany, 1966), Academic Press, New York, 1968, pp. 77-81.

[5] J. Komlós and M. Simonovits, Szemerédi's regularity lemma and its applications in graph theory, Combinatorics, Paul Erdős is eighty, Vol. 2 (Keszthely, 1993), Bolyai Soc. Math. Stud., vol. 2, János Bolyai Math. Soc., Budapest, 1996, pp. 295-352.

[6] V. Nikiforov and C.C. Rousseau, Large generalized books are p-good, J. Combin. Theory Ser. B 92 (2004), 85-97.

[7] Vladimir Nikiforov, Stability for large forbidden subgraphs, J. Graph Theory 62 (2009), no. 4, 362-368.

[8] M. Simonovits, A method for solving extremal problems in graph theory, stability problems, Theory of Graphs (Proc. Colloq., Tihany, 1966), Academic Press, New York, 1968, pp. 279-319.

[9] P. Turán, Eine Extremalaufgabe aus der Graphentheorie, Mat. Fiz. Lapok 48 (1941), 436-452. 